\title{
Bayesian Corner Detection
}

\author{
Xining Zhang and Robert M. Haralick \\ Intelligent Systems Laboratory \\ Department of Electrical Engineering, FT-10 \\ University of Washington \\ Seattle, WA 98195
}

\begin{abstract}
Corners play important roles in high level image understanding. They are the main features in many $2 \mathrm{D}$ or $3 \mathrm{D}$ image models associated with image understanding algorithms. The Bayesian corner detection method inputs a sequence of row-column pairs along an arc and outputs the corner positions and the corner included angles that maximize the a posteriori probability. Experiments on artificially generated sequences permit the measurement of errors of the estimated corner positions and included angles versus different noise perturbations, angles and line lengths respectively.
\end{abstract}

\section{Introduction}

Corner detection can be divided into two kinds. One kind detects corners directly from the gray scale image. The other detects corners from an ordered sequence which can be the results of low level image operations. An ordered sequence is a set of row-column pairs, which represents the successive coordinates along an arc. It is this kind of corner detection that we discuss in this paper.

From the point view of arc segmentation, corner detection is a way to segment arcs according to the detected breaking points. Many papers discuss arc segmentation and corner detection[1-11]. The techniques include iterative end point fitting and splitting, tangent angle deflection, prominence, or high curvature. Corners are those points which separate the sequence into subsequences in which each is a maximal sequence coming from a given model such as a line or a curve of specified form. The end points of the subsequences are called corner points, breaking points or dominant points and once found can be employed in polygon detection or polygon matching.

\section{Motivation and Theory}

We are given an observed sequence $S=<\left(\hat{r}_{i}, \hat{c}_{i}\right), i=1, \ldots, K>$, where $\left(\hat{r}_{k}, \hat{c}_{k}\right)$ is the row-column pair within the specified domain $Z_{r} \times Z_{c}$. For the sake of simplicity of our initial description, we assume that the sequence $S$ consists of two contiguous subsequences $S_{1}=<\left(\hat{r}_{1}, \hat{c}_{1}\right), \ldots,\left(\hat{r}_{k}, \hat{c}_{k}\right)>$ and $S_{2}=<\left(\hat{r}_{k+1}, \hat{c}_{k+1}\right), \ldots,\left(\hat{r}_{K}, \hat{c}_{K}\right)>$, arising from two arcs in a specified class. The corner can be estimated by determining the index $k$ and the included corner angle $\theta$ maximizing 


$$
=\frac{W_{1}}{\sqrt{2 \pi} \sigma} \int_{r} e^{-\frac{\left(\dot{r}_{i}-r\right)^{2}}{2 \sigma^{2}}} d r .
$$

Therefore,

$$
\begin{aligned}
P\left(S_{1} \mid \phi_{1}, \rho_{1}\right) & =\prod_{i=1}^{k}\left(P\left(\hat{c}_{i} \mid \hat{r}_{i}, \phi_{1}, \rho_{1}\right) P\left(\hat{r}_{i} \mid \phi_{1}, \rho_{1}\right)\right) \\
& =V_{1} \prod_{i=1}^{k} e^{-\frac{1}{2\left(1+b_{1}^{2}\right) \sigma^{2}}\left(\hat{c}_{i}-a_{1}-b_{1} \hat{r}_{i}\right)^{2}} \prod_{i=1}^{k} \int_{r} e^{-\frac{\left(\hat{r}_{i}-r\right)^{2}}{2 \sigma^{2}}} d r,
\end{aligned}
$$

where

$$
V_{1}=\left(\frac{W_{1}}{2 \pi \sigma^{2} \sqrt{1+b_{1}^{2}}}\right)^{k} .
$$

The probability distribution of the second line on condition that $\phi_{1}$ and $\theta$ are given can be integrated on $\rho_{1}$ :

$$
\begin{aligned}
P\left(S_{2} \mid \phi_{1}, \theta\right) & =\int_{\rho_{2}} P\left(\rho_{2}, S_{2} \mid \phi_{1}, \theta\right) d \rho_{2}, \\
& =\int_{\rho_{2}} P\left(S_{2} \mid \rho_{2}, \phi_{1}, \theta\right) P\left(\rho_{2} \mid \phi_{1}, \theta\right) d \rho_{2} \\
& =\int_{\rho_{2}} P\left(S_{2} \mid \rho_{2}, \phi_{1}, \theta\right) P\left(\rho_{2}\right) d \rho_{2} .
\end{aligned}
$$

The probability $P\left(\rho_{2}\right)$ can be associated with a constant $W_{\rho_{2}}$ if $\rho_{2}$ is assumed uniformly distributed on its domain. Then

$$
\begin{aligned}
P\left(S_{2} \mid \phi_{1}, \theta\right) & =W_{\rho_{2}} \int_{\rho_{2}} P\left(S_{2} \mid \rho_{2}, \phi_{1}, \theta\right) d \rho_{2} \\
& =W_{\rho_{2}} \int_{\rho_{2}} P\left(S_{2} \mid \rho_{2}, \phi_{2}\right) d \rho_{2},
\end{aligned}
$$

where $\phi_{2}=\phi_{1}+\theta$. In a similar computation as that for $P\left(S_{1} \mid \phi_{1}, \rho_{1}\right)$, we can obtain

$$
\begin{aligned}
P\left(S_{2} \mid \rho_{2}, \phi_{2}\right) & =\prod_{i=k+1}^{K}\left(P\left(\hat{c}_{i} \mid \hat{r}_{i}, \phi_{2}, \rho_{2}\right) P\left(\hat{r}_{i} \mid \phi_{2}, \rho_{2}\right)\right) \\
& =V_{2} \prod_{i=k+1}^{K} e^{-\frac{1}{2\left(1+b_{2}^{2}\right) \sigma^{2}}\left(\hat{c}_{i}-a_{2}-b_{2} \hat{r}_{i}\right)^{2}} \prod_{i=k+1}^{K} \int_{r} e^{-\frac{\left(\hat{r}_{i}-r\right)^{2}}{2 \sigma^{2}}} d r
\end{aligned}
$$

where

$$
V_{2}=\left(\frac{W_{2}}{2 \pi \sigma^{2} \sqrt{1+b_{2}^{2}}}\right)^{K-k},
$$




$$
P(k, \theta \mid S) ; k=1, \ldots, K, \theta \in[-\pi, \pi] .
$$

where $k$ is the index indicating the corner location and $\theta$ is the included angle between the two contiguous subsequences at the $k$ th location.

A two line segment sequence consists of two subsequence, $S_{1}$ and $S_{2}$, with respective line angles of $\phi_{1}$ and $\phi_{2}$, where the $\phi_{1}$ is the counterclockwise angle between the first line and the row axis and $\phi_{2}$ is the counterclockwise angle between the second line and the row axis. The included corner angle $\theta$ is given by $\phi_{2}=$ $\phi_{1}+\theta$. The domain of $\phi_{j} ; j=1,2$ is defined over $[-\pi / 2, \pi / 2]$. Let $\rho_{1}$ be the distance between the line and the origin. $P(S \mid k, \theta)$ can be expressed as follows:

$$
\begin{aligned}
P(S \mid k, \theta) & =\iint_{\rho_{1}} P\left(\phi_{\phi_{1}}, \rho_{1}, S \mid k, \theta\right) d \phi_{1} d \rho_{1}, \\
& =\iint_{\rho_{1}} \int_{\phi_{1}} P\left(S_{1} \mid \phi_{1}, \rho_{1}\right) P\left(S_{2} \mid \phi_{1}, \theta\right) P\left(\phi_{1}\right) P\left(\rho_{1}\right) d \phi_{1} d \rho_{1} .
\end{aligned}
$$

Suppose that the noise perturbation model for $\left(\hat{r}_{i}, \hat{c_{i}}\right)$ is $\hat{r}_{i}=r_{i}+\xi_{i}$ and $\hat{c}_{i}=c_{i}+\eta_{i}$, where $\xi_{i}$ and $\eta_{i}$ are independent and identically distributed Gaussian noise with a zero mean and a variance of $\sigma$. Then

$$
P\left(S_{1} \mid \phi_{1}, \rho_{1}\right)=P\left(\left(\hat{r}_{1}, \hat{c}_{1}\right), \ldots,\left(\hat{r}_{k}, \hat{c}_{k}\right) \mid \phi_{1}, \rho_{1}\right)=\prod_{i=1}^{k} P\left(\left(\hat{r}_{i}, \hat{c}_{i}\right) \mid \phi_{1}, \rho_{1}\right) .
$$

where, $P\left(\left(\hat{r_{i}}, \hat{c_{i}}\right) \mid \phi_{1}, \rho_{1}\right)=P\left(\hat{c_{i}} \mid \hat{r_{i}}, \phi_{1}, \rho_{1}\right) P\left(\hat{r_{i}} \mid \phi_{1}, \rho_{1}\right)$. Next, we explore the two conditional probabilities.

$$
P\left(\hat{c_{i}} \mid \hat{r}_{i}, \phi_{1}, \rho_{1}\right)=\frac{1}{\sqrt{2 \pi\left(1+b_{1}\right)} \sigma} e^{-\frac{\left.\mid \hat{c}_{i}-\left(a_{1}+b_{1}^{2} r_{i}\right)\right]^{2}}{2\left(1+b_{1}^{2}\right) \sigma^{2}}}
$$

where $a_{1}$ and $b_{1}$ are the line parameters of the first line for the representation of $c_{i}=a_{1}+b_{1} r_{i}$. They can be obtained from the given line determined from the first line angle $\phi_{1}$ and the line location $\rho_{1}$, which limit the line points $\left(r_{i}, c_{i}\right)$ by the equality constraint $-r_{i} \sin \phi_{1}+c_{i} \cos \phi_{1}=\rho_{1}$.

Since we do not know the true value of $r_{i}$ which should reflect the observed value $\hat{r}_{i}$ in the case of the given line model $\left(a_{1}, b_{1}\right)$, then we have to integrate over the $r$ domain defined as $[0,128]$ :

$$
\begin{aligned}
P\left(\hat{r}_{i} \mid \phi_{1}, \rho_{1}\right) & =\int_{r} P\left(r, \hat{r}_{i} \mid \phi_{1}, \rho_{1}\right) d r \\
& =\int_{r} P\left(\hat{r}_{i} \mid r, \phi_{1}, \rho_{1}\right) P\left(r \mid \phi_{1}, \rho_{1}\right) d r .
\end{aligned}
$$

Assume that $r$ is uniformly distributed along the line model $\left(a_{1}, b_{1}\right)$, then we can express $P\left(r \mid \phi_{1}, \rho_{1}\right)$ by a constant $W_{1}$.

$$
P\left(\hat{r}_{i} \mid \phi_{1}, \rho_{1}\right)=W_{1} \int_{r} P\left(\hat{r}_{i} \mid r, \phi_{1}, \rho_{1}\right) d r
$$


and $W_{2}$ is an associated constant for $P\left(r \mid \phi_{2}, \rho_{2}\right)$ under the assumption that $r$ is uniformly distributed along the line model $\left(a_{2}, b_{2}\right) . a_{2}$ and $b_{2}$ can be determined from $\phi_{2}$ and $\rho_{2}$ in a similar way as that computation of $a_{1}$ and $b_{1}$ from $\phi_{1}$ and $\rho_{1}$.

Furthermore, we assume $\phi_{1}$ to be uniformly distributed on $[-\pi / 2, \pi / 2]$, so that $P\left(\phi_{1}\right)=1 / \pi$, expressed in a constant $W_{\phi_{1}}$. If the involved sequence is produced from an image where $\rho_{1}$ is assumed uniformly distributed on the image domain, then $P\left(\rho_{1}\right)$ is a constant $W_{\rho_{1}}$.

Therefore, the probability

$$
P(S \mid k, \theta)=U \iint_{\rho_{1}} \int_{\phi_{1}} g_{1}\left(\phi_{1}, \rho_{1}\right) g_{2}\left(\phi_{1}, \theta\right) d \phi_{1} d \rho_{1},
$$

where

$$
\begin{aligned}
& U=W_{\rho_{1}} W_{\rho_{2}} W_{\phi_{1}} V_{1} V_{2} V_{3} \\
& V_{3}=\prod_{i=1}^{K} \int_{r} e^{-\frac{\left(r_{i}-r\right)^{2}}{2 \sigma^{2}}} d r \\
& g_{1}\left(\phi_{1}, \rho_{1}\right)=e^{-\frac{1}{2\left(1+b_{1}^{2}\right) \sigma^{2}} \sum_{i=1}^{k}\left(\hat{c}_{i}-a_{1}\left(\phi_{1}, \rho_{1}\right)-b_{1}\left(\phi_{1}, \rho_{1}\right) r_{i}\right)^{2}}, \\
& g_{2}\left(\phi_{1}, \theta\right)=\int_{\rho_{2}} e^{-\frac{1}{2\left(1+b_{2}^{2}\right) \sigma^{2}} \sum_{i=k+1}^{K}\left(\hat{\left.c_{i}-a_{2}\left(\phi_{1}, \theta, \rho_{2}\right)-b_{2}\left(\phi_{1}, \theta, \rho_{2}\right) \hat{r}_{i}\right)^{2}}\right.} d \rho_{2} .
\end{aligned}
$$

\section{A. $P(k, \theta)$ Constant}

Assume $k$ and $\theta$ to be independently uniformly distributed on $[1, K]$ and $[-\pi, \pi]$ respectively, then $P(k, \theta)$ can be associated with a constant $1 / 2 \pi K$.

Therefore, the $k$ and $\theta$ can be determined by maximizing the following computation over the domain of $\phi_{1}$ and $\rho_{1}$,

$$
\iint_{\rho_{1}} g_{\phi_{1}} g_{1}\left(\phi_{1}, \rho_{1}\right) g_{2}\left(\phi_{1}, \theta\right) d \phi_{1} d \rho_{1}
$$

\section{B. $P(k, \theta)$ Non-constant}

Assume random variable $k$ and $\theta$ to be independent. Therefore, we have

$$
P(k, \theta)=P(k) P(\theta) \text {. }
$$

Assume $k$ to be uniformly distributed along the sequence with the length of $K$, then its corresponding p.d.f.

$$
P(k)=\frac{1}{K} \text {. }
$$

Assume $\theta$ be normally distributed around a $\theta_{0}$, then its corresponding p.d.f.

$$
P(\theta)=\frac{1}{\sqrt{2 \pi} \sigma_{\theta}} e^{-\frac{\left(\theta-\theta_{\theta}\right)^{2}}{2 \sigma_{\theta}^{2}}} .
$$

Therefore, the $k$ and $\theta$ can be determined by maximizing

$$
P(k, \theta) \iint_{\rho_{1}} g_{\phi_{1}} g_{1}\left(\phi_{1}, \rho_{1}\right) g_{2}\left(\phi_{1}, \theta\right) d \phi_{1} d \rho_{1}
$$




\section{Experimental Protocol and Results}

\section{Experimental Protocol}

The experiment measured the statistical distance between the estimated and the true corner position and included angle with the assumption of $P(k, \theta)$ constant. We limit our experiments to the two line segment model for the verification of the theory.

Two line segment sequences can be generated by the following three steps:

(1) Generate an ideal data sequence by specifying the starting point $\left(r_{1}, c_{1}\right)$, the first line length $L_{1}$ and the second line length $L_{2}$, the first line angle $\phi_{1}$ and the included angle $\theta$. In this step, for each line $L_{1}$ or $L_{2}$, if $\left|\cos \phi_{j}\right| \geq\left|\sin \phi_{j}\right|$ $; j=1,2$, we sample the data by increasing the row coordinate by unit steps, otherwise, by increasing the column coordinate by unit steps. After sampling, there results a sequence of $K$ points whose true corner index is $k^{t}$. For the first ideal line generation, if $\left|\cos \phi_{1}\right| \geq\left|\sin \phi_{1}\right|$, then $S_{1}=<\left(r_{i}, c_{i}\right) \mid r_{i}=r_{1}+i ; c_{i}=$ $c_{1}+i \tan \left(\phi_{1}\right)>; i=1, \ldots, k^{t}-1$, where $k^{t}=\left\lfloor L_{1} \cos \phi_{1}\right\rfloor+1$ otherwise, $S_{1}=<$ $\left(r_{i}, c_{i}\right) \mid r_{i}=r_{1}+i \cot \left(\phi_{1}\right) ; c_{i}=c_{1}+i>; i=1, \ldots, k^{t}-1$, where $k^{t}=\left\lfloor L_{1} \sin \phi_{1}\right\rfloor+1$. For the second ideal line generation, if $\left|\cos \phi_{2}\right| \geq\left|\sin \phi_{2}\right|$, then, $S_{2}=<\left(r_{i}, c_{i}\right) \mid r_{i}=$ $L_{1} \cos \phi_{1}+i-k^{t} ; c_{i}=L_{1} \sin \phi_{1}+\left(i-k^{t}\right) \tan \left(\phi_{2}\right)>; i=k^{t}+1, \ldots, K-1$, where $K=$ $k^{t}+\left\lfloor L_{2} \cos \phi_{2}\right\rfloor+1$, otherwise, $S_{2}=<\left(r_{i}, c_{i}\right) \mid r_{i}=L_{1} \cos \phi_{1}+\left(i-k^{t}\right) \cot \left(\phi_{2}\right) ; c_{i}=$ $L_{1} \sin \phi_{1}+i-k^{t}>, i=k^{t}+1, \ldots, K-1$, where $K=k^{t}+\left\lfloor L_{2} \sin \phi_{2}\right\rfloor+1$.

(2) Generate two Gaussian noise sequences $<m_{1 i} ; i=1, . ., K>$ and $<m_{2 i} ; i=$ $1, . ., K>$, where each $m_{j i} ; j=1,2 ; i=1, \ldots, K$ is an independent random variable coming from a Gaussian distribution with zero mean and a standard deviation $\sigma$.

(3) Obtain a perturbed sequence $\left.<\left(r_{i}+m_{1 i}, c_{i}+m_{2 i}\right) ; i=1, \ldots, K\right\rangle$. Since the sequence may violate the ordering constraints, we have to sort the sequence to obtain the ordered sequence $\left\langle\left(\hat{r}_{i}, \hat{c}_{i}\right) ; i, \ldots, K\right\rangle$ desired for the perturbation. If $\left|\cos \phi_{j}\right| \geq\left|\sin \phi_{j}\right| ; j=1,2$, the sorting is done according to the row coordinate, otherwise, on the column coordinate. Then we get the perturbed sequence $S=<$ $\left(\hat{r}_{i}, \hat{c}_{i}\right) \mid i=1, \ldots, K>$ in which the true corner position index $k^{t}$ and the included true corner angle $\theta^{t}$ are contained. For each specified noise standard deviation $\sigma$, we repeat the experiment for $N_{\text {run }}$ times of runs.

In this experiment, $L_{1}=50, L_{2}=50,\left(r_{1}, c_{1}\right)=(0.0,0.0), \phi_{1}=30^{\circ}, \theta=$ $90^{\circ}, \sigma=\{0.4,0.6,0.8,1.0,1.2,1.4,1.6,2.0,2.5,3.0\}$ and $N_{\text {run }}=\{20,40,60,80,100\}$.

Let $\left\{k_{j}, j=1, \ldots, N_{\text {run }}\right\}$ be the set of indices of estimated corner positions and $\left\{\theta_{j}, j=1, \ldots, N_{\text {run }}\right\}$ be the set of estimated included angles determined from the Bayesian corner detector. For each run, we can obtain the error of the estimated corner position,

$$
d_{p j}=\sqrt{\left(\hat{r}\left(k_{j}\right)-r\left(k^{t}\right)\right)^{2}+\left(\hat{c}\left(k_{j}\right)-c\left(k^{t}\right)\right)^{2}},
$$

and the error of the included corner angle

$$
d_{\theta j}=\left|\theta_{j}-\theta^{t}\right| \text {. }
$$

Then, we can obtain the means of the these errors by

$$
\begin{aligned}
& \bar{d}_{p}=\frac{1}{N_{\text {run }}} \sum_{j=1}^{N_{\text {run }}} d_{p j}, \\
& \bar{d}_{\theta}=\frac{1}{N_{\text {run }}} \sum_{j=1}^{N_{\text {ru } n}} d_{\theta j} .
\end{aligned}
$$


and the variances of the these errors by

$$
\begin{aligned}
& \operatorname{var}\left(d_{p}\right)=\frac{1}{\left(N_{\text {run }}-1\right)} \sum_{j=1}^{N_{r u n}}\left(d_{p j}-\bar{d}_{p}\right)^{2}, \\
& \operatorname{var}\left(d_{\theta}\right)=\frac{1}{\left(N_{\text {run }}-1\right)} \sum_{j=1}^{N_{r u n}}\left(d_{\theta j}-\bar{d}_{\theta}\right)^{2} .
\end{aligned}
$$

\section{Experimental Results}

Figure 1 shows the error between the estimated corner position and the true corner position with respect to the different noise standard deviation. For this experiment, number of runs $N_{\text {run }}$ is 100 . This plot indicates that estimated corner position errors are relatively small and stable for the small noise perturbation.

Figure 2 shows the error between the estimated included corner angle and the true included corner angle with respect to the noise standard deviation. This figure has the same meaning as the figure 1 .

Figure 3 shows the error of the included corner angle versus the different corner index $k$. This plot indicates that the estimated corner angle has smaller error when the true corner is located in the middle of the sequence.

\section{Further Work}

We are currently applying the theory described in this paper to detect the dominant point for the two line segment model sequence. Our aim is to apply this method to the dominant points for the multiple line segment model sequence and then for a more arbitrary curve model sequence. In the general case, we assume the given sequence contains multiple subsequences which arise line segments or any other given curve model. We assume that the angle distribution $P(\theta)$ of the included angle between two segments is given and the number of curve segments is $N+1$, then there will be $N$ corners distributed along the sequence $S=\left\langle\left(\hat{r}_{i}, \hat{c}_{i}\right), i=1, \ldots K\right\rangle$ and the sequence will be partitioned into subsequences $S_{i}, i=1, \ldots, N+1$ with respective lengths of $k_{i}, i=1, \ldots, N+1$ and line angles of $\phi_{i}, i=1, \ldots, N+1$, where $\phi_{i}$ is the angle between the line $S_{i}$ and row coordinate. $\theta_{i}=\phi_{i+1}-\phi_{i} ; i=1, \ldots, N$.

The corners can be obtained by maximizing the posterior probability distribution $P\left(k_{1}, \ldots, k_{N} ; \theta_{1}, \ldots, \theta_{N} \mid S\right)$. According to the Bayes' theorem, the posterior probability distribution is proportional to

$$
P\left(S \mid k_{1}, \ldots, k_{N} ; \theta_{1}, \ldots, \theta_{N}\right) P\left(k_{1}, \ldots, k_{N} ; \theta_{1}, \ldots, \theta_{N}\right),
$$

where $P\left(k_{1}, \ldots, k_{N} ; \theta_{1}, \ldots, \theta_{N}\right)$ contains the prior information about the distribution of $k_{1}, \ldots, k_{N} ; \theta_{1}, \ldots, \theta_{N}$, and $P\left(S \mid k_{1}, \ldots, k_{N} ; \theta_{1}, \ldots, \theta_{N}\right)$ reflects the likelihood of the sequence $S$ coming from the assumed model $\left(k_{1}, \ldots, k_{N} ; \theta_{1}, \ldots, \theta_{N}\right)$.

We will also work on the performance characterization of this method by a combination of theoretical analysis and experimental observation. The performance characterization will concentrate on the analysis method and test with a large groups of simulated noisy data[11,12]. After the simulation of synthetic data, the detection method should be applied to process sequences generated from real images. 


\section{Conclusions}

We have discussed the theory and experiments of a Bayesian corner detection method. The theory was explored for the estimation of the corner position and the included corner angle of the two line segment model. The method, however, can be applied to corner detection in sequences having more than two subsequences. We tested the method by using the synthetic data which reflects the sequence model and the Gaussian noise perturbation onto each sequence element independently. On the bases of our preliminary experiments, we are assured that this method is feasible and applicable for the dominant point detection. As for the multiple corner detection, we here suggest a global optimization method which obtains the best detection by comparing all of estimations related to different number of line segiment on the sequence. It is also possible to recursively apply the two line segment model to a multiple corner sequence.

\section{References}

[1] Anderson, I.M., and J.C. Bexdek, "Curvature and Tangential Deflection of Discrete Arcs: A Theory Based on the Commutator of Scatter Matrix Pairs and Its Application to Vertex Detection in Planar Shape Data," IEEE Transactions on Pattern Analysis and Machine Intelligence, Vol. PAMI-6, No. 1, January 1984 , pp. $27-40$.

[2] Davis, L.S., "Understanding Shape: Angles and Sides," IEEE Transactions on Computers, Vol. C-26, No. 3, 1977, pp. 236-242.

[3] Freeman, H., "Shape Description Via the Use of Critical Points," Pattern Recognition, Vol. 10, 1978, pp. 159-166.

[4] Pavlidis, T., "Waveform Segmentation Through Functional Approximation," IEEE Transactions on Computers, Vol. C-22, No. 7, 1973, pp. 689-697.

[5] Phillips, T.Y., and A. Rosenfeld, "An ISODATA Algorithm for Straight Line Fitting," Pattern Recognition Letters, Vol. 7, 1988, pp. 291-297.

[6] Ramer, U., "An Iterative Procedure for the Polygonal Approximation of Plane Curves," Computer Graphics and Image Processing, Vol. 1, 1972, pp. 244-256.

[7] Rosenfeld, A., and E. Johnston, "Angle Detection on Digital Curves," IEEE Transactions on Computers, 1973, pp. 875-878.

[8] Shirai, Y., "A Context Sensitive Line Finder for Recognition of Polyhedra," Artificial Intelligence, Vol. 4, 1973, pp. 95-119.

[9] Shirai, Y., "Edge Finding, Segmentation of Edges and Recognition of Complex Objects," Fourth International Joint Conference on Artificial Intelligence, Los Altos, CA, 1975, pp. 674-681.

[10] Teh, C.-H., and R.T. Chin, "A Scale-Independent Dominant Point Detection Algorithm,"in Proceedings, IEEE Computer Society Conference on Computer Vision and Pattern Recognition, Ann Arbor, Michigan, 1988, pp. 229-234.

[11] Robert M. Haralick, and Tsaiyun Phillips, "Subpixel Precision Corner Detection and Localization," 
[12] Robert M. Haralick, "Performance Characterization in Image Analysis: Thinning, A Case in Point", Pattern Recognition Letters 13, 1992, pp. 5-12.

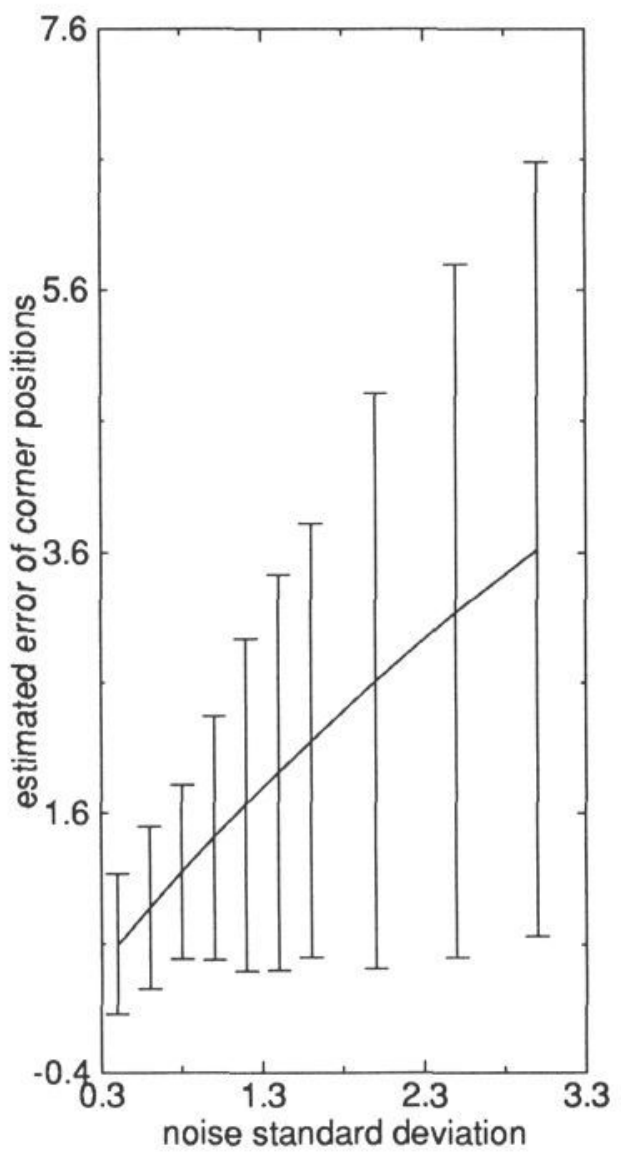

Figure 1: Corner position errors vs. noise perturbation $(\sigma)$, where $\theta=90^{\circ}$, $L_{1}=L_{2}=50, N_{\text {run }}=100 \times 10$. 


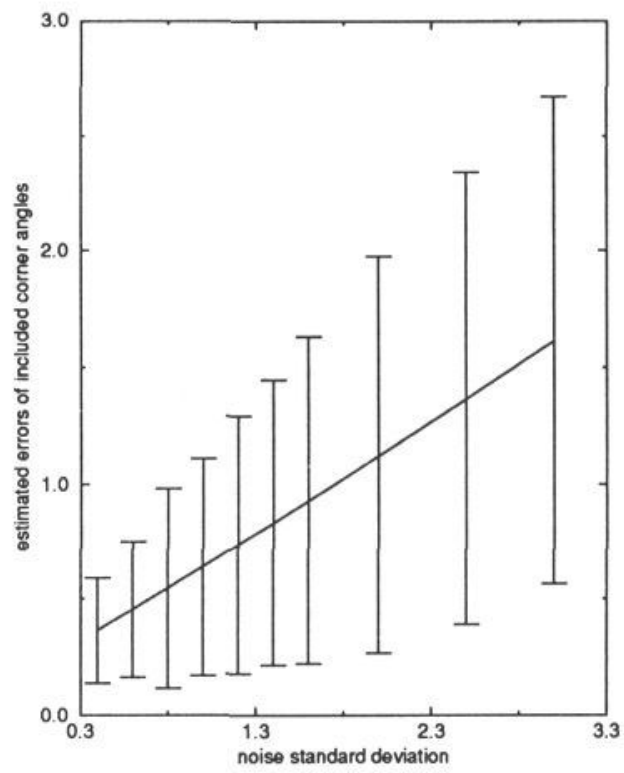

Figure 2: Corner angle errors vs. noise perturbation $(\sigma)$, where $\theta=90^{\circ}, L_{1}=$ $L_{2}=50, N_{\text {run }}=100 \times 10$.

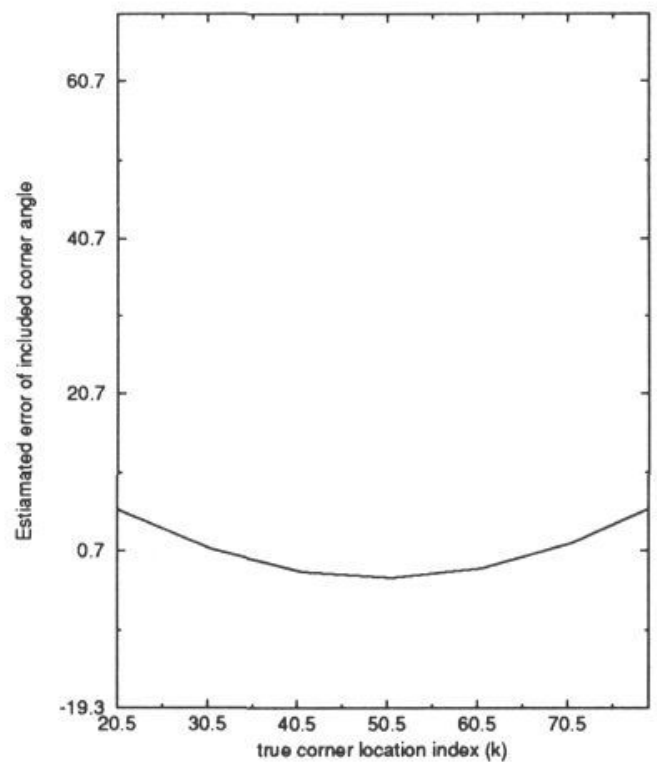

Figure 3: Errors of included corner angles vs. corner index $(k)$, where $\sigma=0.8$, $L_{1}=L_{2}=50, N_{\text {run }}=100$. 
